\title{
Percutaneous endovascular repair of acute type A intramural hematoma of the ascending aorta
}

\author{
Ali Khoynezhad \\ MemorialCare Heart and Vascular Institute, Long Beach, CA, USA \\ Correspondence to: Ali Khoynezhad, MD, PhD, FACS. Director of Cardiovascular Surgery, MemorialCare Long Beach Medical Center, 2801 Atlantic \\ Ave, Long Beach, CA 90806, USA. Email: akhoynezhad@memorialcare.org.
}

Submitted Apr 30, 2019. Accepted for publication Jul 25, 2019.

doi: 10.21037/acs.2019.08.03

View this article at: http://dx.doi.org/10.21037/acs.2019.08.03

\section{Clinical vignette}

An 88-year-old female with history of chronic obstructive pulmonary disease (COPD), renal insufficiency, hyperlipidemia, hypertension and known coronary artery disease with a previous myocardial infarction (MI) was admitted with hypertensive urgency and chest pain. A recurrent MI was ruled out in the intensive care unit (ICU). The patient had a sudden episode of hypotension requiring cardiac resuscitation. Echocardiography was unremarkable, and a subsequent computed tomography aortogram (CTA) revealed an ascending aortic intramural hematoma with a pericardial effusion. Her iliofemoral vessels were able to accommodate the passage of a delivery sheath. Due to her co-morbidities, she was not an open surgical candidate. After discussing risks and benefits, a transfemoral thoracic endovascular repair (TEVAR) of the intramural hematoma was offered to the patient.

\section{Surgical technique}

\section{Preparation}

The patient was taken to the hybrid operating room, placed in supine position, and underwent general anesthesia with typical invasive monitoring. The patient was prepped from groin to the neck.

\section{Exposition}

The transfemoral approach for ascending TEVAR was performed using ultrasound-guided access and pre-close technique. The patient was heparinized to achieve a target activated clotting time $\geq 250$ seconds.

\section{Operation}

A micropuncture needle and 0.18 " guidewire was used to gain entry into the right common femoral artery. Positioning of the guidewire was confirmed with fluoroscopy. An angiogram excluded high or low needle stick. The wire was upsized to 0.35 " and an 8 -French introducer was used to dilate the tract. Next, two Proglide (Abbott Vascular) access closure devices were used to pre-close the right femoral artery, and an $8 \mathrm{~F}$ sheath was placed into the external right iliac artery. The intravascular ultrasound (IVUS) was advanced over the guidewire through the sheath into the abdominal, descending, transverse and ascending aorta under direct IVUS imaging and fluoroscopy. The IVUS was used to examine the aortic root, ascending aorta and aortic arch as well as to identify the location of the intimal abnormality, extent of intramural hematoma, and the location of the coronary arteries and the innominate artery. Appropriate diameter measurements of the proximal and distal landing zones above the sinotubular junction and proximal to the innominate artery were obtained as well as the distance from sinotubular junction to the innominate artery orifice. An angiogram was not performed due to patient's poor renal function. Using the preoperative CT scan and intraoperative IVUS, an appropriate stent graft was chosen, paying attention not to oversize greater than $10 \%$ relative to proximal landing zone diameter. The $38 \mathrm{~mm} \times 50 \mathrm{~mm}$ Medtronic Valiant stent graft was positioned at $10 \mathrm{~mm}$ above the coronary artery ostia and a second Valiant $38 \times 50$ was used to extend to the 
orifice of the innominate artery. The first aortic stent graft was deployed with administration of $36 \mathrm{mg}$ of intravenous adenosine to minimize cardiac output and ensure precise device deployment. Given the extension of intramural hematoma in the descending thoracic aorta and a possible source of entry in the proximal descending thoracic aorta, a third $32 \mathrm{~mm} \times 150 \mathrm{~mm}$ Valiant stent graft was deployed in the descending thoracic aorta immediately after the take-off the left subclavian artery.

\section{Completion}

Intraoperative transesophageal echocardiogram confirmed proximal positioning of the stent graft at or above the sinotubular junction, adequate function of the aortic valve, no change in cardiac function and no significant increase in pericardial effusion. After completion IVUS assessment to confirm adequate device apposition to the adjacent aorta in the proximal and distal seal zones, and patency of the coronary arteries, the $18 \mathrm{~F}$ introducer sheath was removed and the Proglide sutures were tied down. Heparin was reversed with protamine.

\section{Comments}

\section{Clinical results}

Our group has obtained the first physician-sponsored investigational device exemption (PS-IDE) for using a dedicated stent graft for ascending aortic pathologies in the United States $(1,2)$. We studied eleven patients within this PS-IDE and thirty-two other patients using commercially available stent grafts. A total of eight patients have been treated for type A intramural hematoma with no operative mortality or conversion to open aortic replacement. In the thirty-five months mean follow-up, we had two mortalities due lung cancer and gastrointestinal bleeding (3).

\section{Advantages}

The current endovascular stent graft technology offers lessinvasive alternative treatment option in selected high-risk patients with acute type A intramural hematoma, who are unfit for surgical repair. With improved device design and avoidance of cardiopulmonary bypass or sternotomy, there is likelihood of improved early outcomes using TEVAR when compared to open repair in this high-risk patient population who are anatomically suitable candidates for endovascular repair.

\section{Caveats}

TEVAR for acute type A intramural hematoma may be associated with a spectrum of complications. The most serious complications of TEVAR are neurologic resulting from ischemia or embolization. The proximity of the coronary artery ostia and aortic valve to the proximal landing zone increases the risk of coronary artery coverage or aortic valve dysfunction. Last but not least, the longevity of the stent graft in the ascending aorta is yet to be established.

\section{Acknowledgments}

None.

\section{Footnote}

Conflicts of Interest: The author has no conflicts of interest to declare.

\section{References}

1. Khoynezhad A, Donayre CE, Walot I, et al. Feasibility of Endovascular Repair of Ascending Aortic Pathologies as part of the FDA-Approved Physician SponsoredInvestigational Device Exemption. J Vasc Surg 2016;63:1483-95.

2. Shah A, Khoynezhad A. Thoracic endovascular repair for acute type A aortic dissection: operative technique. Ann Cardiothorac Surg 2016;5:389-96.

3. Horton JD, Kölbel T, Haulon S, et al. Endovascular Repair of Type A Aortic Dissection: Current Experience and Technical Considerations. Semin Thorac Cardiovasc Surg 2016;28:312-7.
Cite this article as: Khoynezhad A. Percutaneous endovascular repair of acute type A intramural hematoma of the ascending aorta. Ann Cardiothorac Surg 2019;8(5):585-586. doi: 10.21037/ acs.2019.08.03 